\title{
Electrophysiological correlates of cognitive inhibition in college students with schizotypal traits
}

\author{
Myung-Sun Kim ${ }^{1 *}$, Sung Hwa Oh${ }^{1}$, Kyoung Mi Jang ${ }^{1}$, Huije Che ${ }^{2}$, Chang-Hwan Im $^{2}$ \\ ${ }^{1}$ Department of Psychology, Sungshin Women's University, Seoul, Korea \\ ${ }^{2}$ Department of Biomedical Engineering, Hanyang University, Seoul, Korea \\ Email: ${ }^{*}$ kimms@,sungshin.ac.kr
}

Received 24 September 2011; revised 27 October 2011; accepted 15 November 2011

\begin{abstract}
Objective: We investigated cognitive inhibition in nonclinical individuals with schizotypal traits using eventrelated potentials. Methods: College students with psychometrically defined schizotypal traits $(n=16)$ and normal controls $(n=15)$ participated. The computerized Stroop task with three types of stimuli, i.e., congruent, incongruent, and neutral words, was used to measure cognitive inhibition. Results: The schizotypaltrait group committed significantly more errors in response to incongruent words than did the control group. The control group showed frontal negativity (FN) of significantly greater amplitude in response to incongruent than to congruent and neutral stimuli, whereas the schizotypal-trait group showed no significant difference in FN amplitude between incongruent and congruent/neutral stimuli at 300 - 400 ms poststimulus. A source localization analysis conducted in different waveforms for incongruent minus congruent conditions at $\mathbf{3 0 0}$ - $\mathbf{4 0 0}$ ms poststimulus showed reduced activation in the left cingulate cortex and in the middle/medial prefrontal cortex in the schizotypal-trait group compared with the control group. The two groups did not differ in the sustained potential amplitudes observed at 550 - 650 ms after stimulus-onset at parietal sites. Conclusions: These results suggest that individuals with schizotypal traits have difficulties in conflict detection and cognitive inhibition, possibly mediated by the cingulate cortex and prefrontal cortex.
\end{abstract}

Keywords: Cognitive Inhibition; Event-Related Potentials; Frontal Negativity; Schizotypal Trait; Stroop Task; Sustained Potential

\section{INTRODUCTION}

Deficits in cognitive inhibition, which is the ability to suppress thoughts or emotions irrelevant to goal-directed

\footnotetext{
*Corresponding author.
}

behavior, has recently received attention as a trait marker of schizophrenia because impaired cognitive inhibition is observed not only in chronic or first-episode patients but also in healthy relatives of patients with schizophrenia $[1,2]$.

The Stroop task [3] has been widely used to measure cognitive inhibition. During this task, color words (e.g., RED) printed in colored ink are presented, and individuals are required to respond to the ink color in which the words are printed. The relationship between the ink color and the word meaning can be congruent (e.g., "RED" printed in red ink), incongruent (e.g., "RED" printed in blue ink), or neutral (e.g., string of letters such as "XXXX" or a non-color word such as "BAG" printed in red ink). In general, the incongruent stimuli elicit a longer response time and more errors relative to the congruent or neutral stimuli, a pattern referred to as the Stroop interference effect [4]. It has been suggested that the Stroop effect is caused by the fast, automatic process of word reading that interferes with the slow, controlled process of color naming [5].

Previous studies investigating cognitive inhibition using the Stroop task in patients with schizophrenia have reported increased interference effects in these patients $[2,6]$. For example, patients with schizophrenia show significantly longer response times [6] and more errors in response to incongruent stimuli than did normal controls $[7,8]$. This increased interference effect has been attributed to a disruption in the inhibitory process [5] or a failure to attend to the task-relevant dimension of the stimulus [9].

Neuroimaging studies have consistently reported increased activation of the anterior cingulate cortex (ACC) and prefrontal cortex (PFC) during the incongruent condition compared with the congruent condition of the Stroop task [10-12]. Furthermore, positive correlations between PFC activation and Stroop task performance have been reported $[13,14]$. ACC neural activity is the monitoring function that detects and signals conflict and thereby triggers the PFC to adopt a proper executivecontrol action [15]. Decreased ACC activation in patients 
with schizophrenia during the Stroop task [7,16,17] and an association between structural disruption of the cingulate cortex and performance on the Stroop task [18] have been observed.

Although neuroimaging studies have revealed the brain regions involved in the Stroop task, the temporal course of activation in the brain regions involved during execution of the Stroop task is still unknown [19]. Event-related potentials (ERPs) have been widely used to investigate cognitive functioning due to the high temporal resolution associated with this technique. Numerous studies have shown that compared with the congruent condition, the incongruent condition of the Stroop task elicits more negative and positive potentials at $300-500$ $\mathrm{ms}$ and 600 - $900 \mathrm{~ms}$ poststimulus, respectively [19,20]. Frontal negativity (FN) (also called N450), usually observed in fronto-central sites at $300-500 \mathrm{~ms}$ after stimulus onset, reflects the detection of cognitive conflicts because FN amplitude is modulated by the ratio of congruent to incongruent stimuli [21]. Normal controls show significantly greater FN amplitudes to incongruent than to congruent or neutral stimuli, whereas patients with schizophrenia show no differences in FN amplitudes between incongruent and congruent stimuli [22,23]. The cortical sources of the FN are the ACC [19,20,24,25] and the PFC [26,27].

Sustained positivity (SP), which is observed around 600 - $900 \mathrm{~ms}$ poststimulus, usually at centro-parietal sites, is also sensitive to the degree of conflict between congruent and incongruent stimuli. The SP amplitude is greater in response to incongruent stimuli than to congruent and neutral stimuli in normal controls $[19,26]$, but in patients with schizophrenia, either no difference in SP amplitude is observed between incongruent and congruent stimuli [23] or SP amplitude is significantly reduced [22]. The functional role of SP is less clear than that of the FN. SP has been associated with conflict resolution [23] or response selection and goal maintenance [28]. The sources of SP are poorly understood, although the PFC has been considered as SP generator [25].

ERPs have been widely used to study cognitive inhibition due to their high temporal resolution, but they offer limited spatial resolution. A number of currentdensity estimation techniques have been developed to determine electrophysiological source locations. Low-resolution electromagnetic tomography (LORETA) [29] is one of the methods widely used for solving the inverse problem because it does not require the assumption of a specific number of sources [30]. The assumption made in LORETA is that the inverse problem can be solved by determining the spatially smoothest current distribution, which is achieved by applying a Laplacian operator to the current density. By making this assumption, a particular current-density distribution can be obtained among the infinite number of solutions to the inverse problem.

Patients with schizotypal personality disorder (SPD) or nonclinical individuals with schizotypal traits represent a promising endophenotypic approach to understanding schizophrenia [31]. Indeed, SPD and schizophrenia share common genetic [32], neuroimaging [33], and neuropsychological [34] abnormalities. Only a few studies have investigated cognitive inhibition in patients with SPD or in nonclinical individuals with schizotypal-traits using the Stroop task, and the results have been inconsistent. Some studies have reported an increased Stroop effect in patients with SPD [35] and in nonpsychiatric adults with delusion-proneness [36], whereas other studies have observed no difference in the Stroop effect between low- and high-schizotypal healthy participants [37].

We investigated cognitive inhibition in nonclinical individuals with psychometrically defined schizotypal traits using the Stroop task and ERPs. We were particularly interested in determining whether individuals with schizotypal traits show an increased Stroop interference effect and if so, whether this increased Stroop effect is reflected by ERP components such as FN and SP. Additionally, we localized the generators underlying cognitive inhibition using standardized low-resolution electromagnetic tomography (sLORETA) incorporated in CURRY v. 6.0 and by using individual magnetic resonance imaging (MRI) as a realistic head model.

\section{METHODS}

\subsection{Participants}

Thirty-one female college students were recruited from a pool of 400 students based on their scores on the Korean version of the Schizotypal Personality Questionnaire (SPQ) $[38,39]$. The schizotypal-trait group $(n=16)$ was composed of those who obtained the highest $5 \%$ of scores on the SPQ [39] (score range; 36 - 51), and the control group $(n=15)$ consisted of those who obtained average $( \pm 1 \mathrm{SD})$ scores on the SPQ (score range; $14 \sim 24$ ). The Structured Clinical Interview for DSM-IV-Non Patient [40] was administered to ensure that none of the participants had a history of psychiatric, medical, or neurological disorders or of drug/alcohol abuse. All participants were right-handed, and none was taking medications at the time of testing. All participants were paid for their participation and provided written informed consent after receiving a complete description of the study. This study was approved by the Sungshin Women's University Institutional Bioethics Review Board.

\subsection{The Stroop Task}

The computerized Stroop task, which consists of three types of stimuli, was administered to measure cognitive inhibition. The congruent stimuli were Korean color 
words (red, green, blue and yellow) written in the color indicated by the word and incongruent stimuli were Korean color words written in colors that differed from the words' meanings. Neutral stimuli were non-color words written in one of the four colored inks. A total of 432 stimuli (144 stimuli for each type) were presented randomly in two blocks, and the participants were required to indicate the ink color in which the words were printed by pressing one of four buttons.

The stimuli were presented for $150 \mathrm{~ms}$ in foveal vision on a computer monitor using E-PRIME (Psychology Software Tools, Inc., Sharpsburg, PA, USA), and each subtended a vertical visual angle of $2.29^{\circ}$ and a horizontal visual angle of $3.43^{\circ}$. A crosshair (+) was displayed on the screen for $700 \mathrm{~ms}$ as a fixation point prior to stimulus presentation, and the interstimulus interval was $1300 \mathrm{~ms}$. The experiment was divided into a color-to-key acquisition phase, a practice phase, and a test phase. The colorto-key acquisition phase was administered to allow participants to rehearse mapping the colors onto fingers and pressing the response buttons. It consisted of 100 trials with string of letters "OOOO" in each of the four colors.

\subsection{Electrophysiological Recording Procedure}

Electroencephalographic activity (EEG) was recorded using a 64-channel Geodesic sensor net connected to a 64-channel, high-input impedance amplifier (Net Amp 300: Electrical Geodesics, Eugene, OR, USA) in an electrically shielded and soundproofed experimental room. Each electrode was referenced to $\mathrm{Cz}$, and individual electrodes were adjusted until impedances were less than $50 \mathrm{k} \Omega$ [41]. Eye movements and blinks were monitored with electrodes placed near the outer cantus and beneath the left eye.

During the experiment, EEG activity was recorded continuously using a $0.1-100 \mathrm{~Hz}$ analogue bandpass and a sampling rate of $250 \mathrm{~Hz}$. After data collection was completed, the EEG was segmented into $1000 \mathrm{~ms}$ epochs (including a $100 \mathrm{~ms}$ prestimulus baseline) with respect to the event markers. Epochs contaminated by artifacts such as eye blinks and eye movements were rejected before averaging (the threshold for artifact rejections was \pm 70 $\mu \mathrm{V})$. Data obtained from the Stroop task were then averaged for each subject and each stimulus type (congruent, incongruent, and neutral). An average-reference transformation was used to minimize the effects of referencesite activity [42]. ERPs were baseline-corrected with respect to the $100 \mathrm{~ms}$ prestimulus baseline recording interval and were digitally low-pass filtered at $30 \mathrm{~Hz}$. Only those EEGs associated with correct responses were subjected to statistical analyses.

\subsection{Estimation of Current Densities Using SLORETA}

High-resolution T1-weighted MRIs were obtained from all participants using a Philips 3T scanner (Philips Intera, Philips Medical System, Best, the Netherlands). The scalp location of each electrode was determined with a FASTRAK 3D-digitizer (Polhemus Inc., VT, USA). The electrode locations were imported into the Curry v. 6.0 software, where the MRIs and electrode locations of the subjects were spatially co-registered for source localization. Three points, the nasion and left and right preauricular points, were used to match each sensor location with individual MRIs. A three-compartment boundaryelement model was computed for each participant. The resolution of the meshes was set to 9,8 , and $6 \mathrm{~mm}$ for skin, skull, and brain, respectively. After segmenting the gray matter of the brain, a representation of the cortex excluding the brainstem and cerebellum was computed to limit the source space for the inverse solution. We reconstructed the current-density distribution on each individual's cortex using the sLORETA algorithm [43] implemented in CURRY v. 6.0, which evaluates statistical source images by performing an inverse weighting of the LORETA imaging results and their estimated variances. The regularization parameter was automatically determined by the $\chi^{2}$ criterion method implemented in CURRY. We conducted the sLORETA analysis at the time point of the peak mean global field power (MGFP) in different waveforms for incongruent minus congruent conditions at $300-400 \mathrm{~ms}$ poststimulus. We used the statistical parametric mapping toolbox (SPM8) (http:// www.l.ion.ucl.ac.uk/spm/) implemented in Matlab version 7.1 (Mathworks, USA) for the current-density-image analysis. We applied realigning, coregistering, normalizing, and smoothing for the spatial preprocessing of the current-density image [44]. Finally, statistical parametric mapping was applied to the normalized current-density images using SPM8 and the two-sample $t$-test to statistically compare the current density elicited by the incongruent minus congruent conditions.

\subsection{Statistical Analysis}

Based on the grand-average and individual ERP waveforms, two ERP components were selected. One component, FN, was a phasic negative peak observed at 300 $400 \mathrm{~ms}$ poststimulus, mainly at frontal sites, and the other component was sustained positive potentials (SP) observed at 550 - $650 \mathrm{~ms}$ mainly, at parietal sites. The mean amplitudes at $300-400 \mathrm{~ms}$ and at $550-650 \mathrm{~ms}$ for each stimulus type were calculated and analyzed with a repeated measures, mixed-design analysis of variance (ANOVA) with electrode sites (Fz, F3, F4 for FN and Pz, P3, and $\mathrm{P} 4$ for SP) and stimulus type (congruent, incongruent, and neutral stimuli) as within-subject factors and group (schizotypal-trait group and control group) as the between-subject factor. Greenhous-Geisser corrections for multiple comparisons were employed when appropriate, 
and the corrected $p$ values are reported. Variables showing significant main effects were further analyzed using a paired $t$-test or a one-way ANOVA. A regression analysis with cortical activation (incongruent minus congruent condition) as independent and performances on the Stroop task (response time and accuracy) as dependent variables was conducted.

The response time and accuracy were subjected to a repeated measures, mixed-design ANOVA, with stimulus type as the within-subject factor and group as the between-subject factor. The demographic characteristics of the participants in the schizotypal and control groups were compared using a one-way ANOVA.

\section{RESULTS}

\subsection{Demographic Characteristics}

The analysis of the demographic data showed that the schizotypal-trait and control groups did not differ in age $\left(F_{1,29}=\right.$ $2.53, n s)$. The mean ages of the schizotypal-trait and control groups were 19.88 years $(\mathrm{SD}=1.26)$ and 21.00 years $(\mathrm{SD}=2.17)$, respectively. However, the SPQ scores of the two groups differed significantly $\left(F_{1,29}=\right.$ $148.11, p<0.0001)$ in that the schizotypal-trait group obtained significantly higher SPQ scores (mean $=41.81, \mathrm{SD}=$ $6.10)$ than did the control group (mean $=17.67, \mathrm{SD}=3.02)$.

\subsection{Behavioral Results of the Stroop Task}

Statistical analysis of the response time (RT) during the Stroop task showed a main effect of stimulus type $\left(F_{2,58}\right.$ $=66.51, p<0.0001)$. The incongruent stimuli elicited significantly longer RTs than did congruent $\left(t_{30}=-8.74\right.$, $p<0.0001)$ and neutral stimuli $\left(t_{30}=-6.99, p<0.0001\right)$. Neutral stimuli elicited longer RT than did congruent stimuli $\left(t_{30}=-9.58, p<0.0001\right)$. The RTs of the schizotypal-trait and control groups were not significantly different $\left(F_{1,29}=0.14, n s\right)$.

In terms of accuracy, we found main effects of stimulus type $\left(F_{2,58}=40.46, p<0.0001\right)$ and group $\left(F_{1,29}=\right.$ $7.87, p<0.01)$. The incongruent stimuli elicited signifycantly more errors than did congruent stimuli $\left(t_{30}=7.46\right.$, $p<0.0001)$, and neutral stimuli elicited more errors than did congruent stimuli $\left(t_{30}=9.60, p<0.0001\right)$. However, no significant difference was found between incongruent and neutral stimuli $\left(t_{30}=-1.88, n s\right)$. Furthermore, the schizotypal-trait group showed significantly more errors, particularly in response to incongruent $\left(F_{1,29}=3.12, p<\right.$ $0.05)$ and neutral stimuli $\left(F_{1,29}=11.53, p<0.01\right)$, but not to congruent stimuli $\left(F_{1,29}=2.25, n s\right)$, than did the normal control group. Mean RTs and accuracy rates for the three stimulus types in the two groups are presented in Table 1.

\subsection{ERP Results on the Stroop Task}

The grand-average ERPs elicited at $\mathrm{Fz}$ and $\mathrm{Pz}$ in response to the three types of stimuli are depicted in Figure 1 for both groups. Incongruent stimuli elicited more negativity (FN) than did congruent and neutral stimuli at $300-400 \mathrm{~ms}$ at $\mathrm{Fz}$ for the control group, but no difference in FN amplitudes was observed between congruent and incongruent stimuli in the schizotypal-trait group.

However, both groups showed more positivity (SP) at $550-650 \mathrm{~ms}$ at $\mathrm{Pz}$ in response to incongruent than that to congruent stimuli. Table 2 summarizes the mean amplitudes of the FN at frontal sites and those of SP at parietal sites observed in the two groups.

Main effects of stimulus type $\left(F_{2,58}=12.59, p<0.0001\right)$ and electrode site $\left(F_{2,58}=7.91, p<0.01\right)$ were observed for FN. Incongruent words elicited larger FN amplitudes than did congruent $(p<0.0001)$ and neutral stimuli $(p<$ $0.0001)$, and the amplitude measured at Fz was larger than that measured at F3 $(p<0.01)$ and F4 $(p<0.0001)$. Additionally, an interaction effect of group by stimulus type $\left(F_{2,58}=3.20, p<0.05\right)$ was identified. In the control group, incongruent words elicited larger $\mathrm{FN}$ amplitude than did congruent $(p<0.001)$ and neutral stimuli $(p<$ $0.0001)$, whereas no significant differences were observed between incongruent and congruent stimuli $\left(t_{15}=\right.$ $-1.66, n s)$ or between incongruent and neutral stimuli $\left(t_{15}\right.$ $=-1.45, n s)$ in the schizotypal-trait group.

The statistical analysis of SP amplitude showed only a main effect of stimulus type $\left(F_{2,58}=11.13, p<0.0001\right)$. The incongruent stimuli elicited larger SP amplitude than did neutral $(p<0.0001)$ and congruent stimuli $(\mathrm{p}<0.0001)$.

Table 1. The mean response time (RT) and accuracy for congruent, incongruent and neutral stimuli of the Stroop task in schizotypaltrait and control groups.

\begin{tabular}{cccccccc}
\hline \multicolumn{3}{c}{ Schizotypal-trait group $(\mathrm{n}=16)$} & \multicolumn{3}{c}{ Control group $(\mathrm{n}=15)$} \\
\hline & Congruent & Incongruent & Neutral & Congruent & Incongruent & Neutral \\
\hline \multirow{2}{*}{ RT (ms) } & 539.24 & 623.78 & 570.38 & 521.55 & 621.89 & 557.16 \\
& $(83.92)$ & $(86.80)$ & $(85.24)$ & $(56.18)$ & $(84.66)$ & $(64.28)$ \\
Accuracy (\%) & & & & & 86.00 & 87.73 \\
& 90.44 & 83.13 & 84.31 & 92.33 & $(3.35)$ & $(1.91)$ \\
\hline
\end{tabular}

SD in parenthesis. 
Table 2. The mean amplitudes of frontal negativity and sustained potential for three types of stimuli of the Stroop task in schizotypal-trait and control groups.

\begin{tabular}{|c|c|c|c|c|c|c|}
\hline & \multicolumn{2}{|c|}{ Schizotypal-trait group $(n=16)$} & \multirow[b]{2}{*}{ Neutral } & \multicolumn{2}{|c|}{ Control group $(n=15)$} & \multirow[b]{2}{*}{ Neutral } \\
\hline & Congruent $(\mathrm{uV})$ & Incongruent & & Congruent & Incongruent & \\
\hline \multicolumn{7}{|l|}{$\mathrm{FN}$} \\
\hline FZ & $-0.72(2.49)$ & $-1.16(2.28)$ & $-0.72(2.83)$ & $-1.08(2.42)$ & $-2.15(2.67)$ & $-22(2.66)$ \\
\hline F3 & $-0.36(2.69)$ & $-0.81(2.30)$ & $-0.30(2.96)$ & $0.31(1.46)$ & $-0.75(1.35)$ & $0.88(1.94)$ \\
\hline $\mathrm{F} 4$ & $-0.29(2.30)$ & $-0.86(1.85)$ & $-0.56(2.20)$ & $-.10(1.88)$ & $-0.80(1.92)$ & $-0.37(2.21)$ \\
\hline \multicolumn{7}{|l|}{ SP } \\
\hline $\mathrm{PZ}$ & $1.45(2.34)$ & $2.70(2.76)$ & $1.70(2.70)$ & $1.09(3.79)$ & $2.21(4.03)$ & $1.07(2.93)$ \\
\hline P3 & $1.41(1.90)$ & $2.57(2.65)$ & $1.75(2.70)$ & $1.20(2.93)$ & $2.02(3.57)$ & $1.44(2.66)$ \\
\hline P4 & $1.01(1.98)$ & $1.78(1.31)$ & $1.53(2.05)$ & $-0.82(1.59)$ & $1.07(1.71)$ & $-0.93(1.42)$ \\
\hline
\end{tabular}

FN: Frontal negativity; SP: Sustained potential, SD in parenthesis.
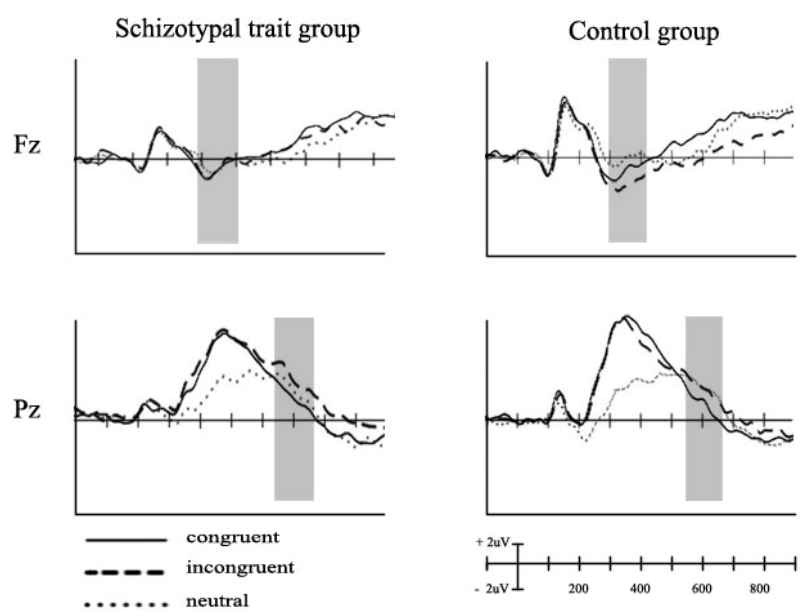

Figure 1. Grand averaged event-related potentials elicited at $\mathrm{Fz}$ and $\mathrm{Pz}$ in response to the three types of stimuli for schizotypaltrait and control groups.

\subsection{Source Localization}

The sLORETA analysis was conducted at the time point of the peak MGFP in different waveforms for incongruent minus the congruent conditions at $300-400 \mathrm{~ms}$ poststimulus. The current density in the left cingulate gyrus (BA 24), medial frontal gyrus (BA 6), and middle frontal gyrus (BA 8) showed significant reductions in the schizotypal-trait group $(\mathrm{T}=2.95, p=0.005$ uncorrected, extent $\mathrm{k}=50$ ) compared with that in the normal control group (Figure 2). The regression analysis showed a significant association between activation of left cingulate gyrus (incongruent minus congruent conditions) and accuracy on incongruent condition of the Stroop task in schizotypal-trait group $(r=0.70, p=0.05)$, but this association was not observed in control group $(r=0.30, n s)$.

The generators underlying SP were not analyzed in this study, as we did not observe a distinct peak at $550-650$ ms poststimulus.

\section{DISCUSSION}

We used the Stroop task and ERPs to investigate whether nonclinical individuals with psychometrically defined schizotypal traits have a deficit in cognitive inhibition. The results of the Stroop task showed that both schizotypal-trait and control groups showed longer RTs and more errors in response to incongruent than to congruent stimuli. However, the schizotypal-trait group committed significantly more errors in response to incongruent stimuli than did the normal control group. These results were consistent with those of previous studies, which reported increased error rates in response to incongruent stimuli in patients with schizophrenia $[7,8,16,45]$ or SPD [35] relative to normal controls.

The increased interference effect observed in patients with schizophrenia has been interpreted as reflecting an impairment in selective attention $[9,46]$ or an inability to inhibit automatic processes such as word reading [5].

(a)
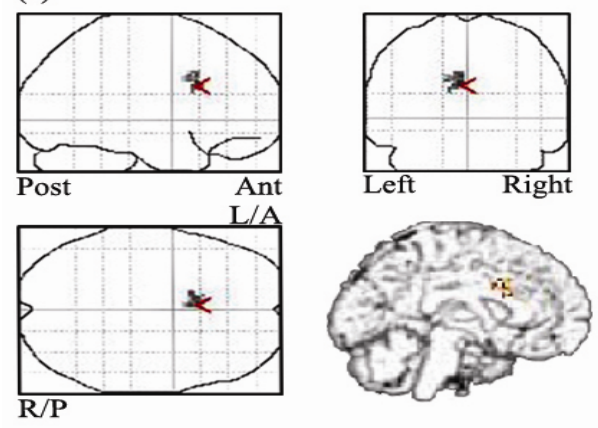

(b)
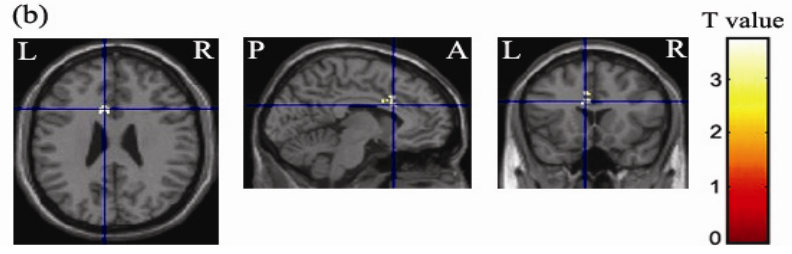

Figure 2. Statistical parametric maps displaying decreased current density at the time point of the peak MGFP of the incongruent minus the congruent conditions at $300-400 \mathrm{~ms}$ poststimulus (a) and map of $t$ statistic $(\operatorname{Spm}\{t\})(b)$. L/A: left anterior, $\mathrm{R} / \mathrm{P}$ : right posterior. Reduced activation in the left cingulate gyrus, medial/middle frontal gyrus was observed in schizotypal-trait group compared with that in the control group. 
Therefore, increased error rates in response to incongruent stimuli in individuals with schizotypal traits suggest that these individuals have difficulties inhibiting irrelevant dimensions and/or allocating attention to relevant stimuli dimensions.

In the present study, the two groups did not show any significant RT differences in response to the three types of stimuli during the Stroop task. We used Korean noncolor nouns as neutral stimuli rather than string of letters such as "XXXX" because "XXXX" could be perceived as an English word by our Korean participants. We found that neutral stimuli elicited significantly longer RTs and more errors than did congruent stimuli, and these results were inconsistent with those of previous studies that found no significant differences between neutral and congruent stimuli in terms of RT [47] or error rate [48]. Because the congruent words were from a single semantic category (color), whereas the neutral words were from more than one semantic category (e.g., BAG, DOG), the neutral words may have been semantically more difficult than congruent words, which led to increased RTs for neutral words and further resulted in the absence of an increased interference effect on RT (the interference effect on RT is usually calculated as incongruent RT minus neutral RT) [4,45]. Some studies have observed an increased RT interference effect only in patients with paranoid subtype or severe psychotic symptoms [49]. Therefore, the absence of an increased RT interference effect may be related to methodological differences such as the particular neutral stimuli and the participants employed in this study.

The control group showed significantly greater FN amplitude in response to incongruent stimuli relative to congruent and neutral stimuli, whereas no significant difference in FN amplitudes was observed between incongruent and congruent stimuli in the schizotypal-trait group. These results are consistent with those of previous studies that observed no significant difference in N450 amplitudes between incongruent and congruent stimuli in patients with schizophrenia [22,23]. The FN or N450 reflects the detection of cognitive conflicts because the FN amplitude is modulated by the ratio of congruent to incongruent stimuli, and FN amplitude elicited by incongruent is greater than that elicited by congruent or neutral stimuli $[21,24]$.

Furthermore, the source-localization analysis of the difference in the waveforms between the incongruent and congruent conditions showed decreased activation of the left cingulate gyrus and medial/middle frontal gyrus at $300-400 \mathrm{~ms}$ poststimulus in the schizotypal-trait group relative to the control group. In addition, there was a significant association between activation of the left cingulate gyrus and accuracy on incongruent condition of the Stroop task in schizotypal-trait group.
Previous studies have reported activation in the ACC [19,20,24,25] and PFC [27] during the Stroop task. Previous neuroimaging studies observed decreased activetion of the ACC during incongruent condition of the Stroop task in patients with schizophrenia [7,16,17]. Additionally, patients with schizophrenia and SPD have structural abnormalities of the ACC and the PFC [50,51,52]. Therefore, the present results indicate that individuals with schizotypal traits have difficulties detecting cognitive conflicts, and this may be mediated by the ACC and/ or PFC.

Both the schizotypal-trait and control groups showed greater SP amplitude in response to incongruent stimuli than that to congruent or neutral stimuli, and the two groups did not differ in terms of SP amplitudes. These results are inconsistent with those of previous studies that observed reduced SP amplitudes in response to incongruent stimuli in patients with schizophrenia relative to normal controls [23]. However, a recent study reported that patients with schizophrenia have reduced SP amplitude in response to incongruent stimuli only in the first block but not in the second block when stimuli are presented in two blocks, indicating that SP amplitude could be affected by practice [22]. Only studies that employed the modified Stroop task, which requires more attention, and included patients with more severe psychotic symptoms show reduced SP amplitude in response to incongruent stimuli [22]. Therefore, the absence of a difference in SP amplitude between the schizotypal-trait and control groups seemed to be due participants' having less severe symptoms and to the classic single-trial version of the Stroop task employed in this study.

Although the functional role of SP is less clear than that of FN, SP reflects a solution to a cognitive conflict [23] or response selection and goal maintenance, as a positive correlation is observed between response accuracy and SP amplitude [28]. However, in the present study, although individuals with schizotypal traits made more errors in response to incongruent stimuli than did the controls, the SP amplitudes did not differ between the two groups. Future study is needed to understand the functional significance of the SP.

Our study had several limitations that should be addressed in future studies. First, the inclusion of only a small number of female participants limits the generalizability of the findings. Second, the use of noncolor words from more than a single semantic category as neutral stimuli prevents us from eliminating the effect of semantics on the results. Therefore, future studies should use neutral words from a single semantic category to understand performance on the Stroop task in individuals with schizospectrum disorders including schizophrenia.

In summary, nonclinical individuals with schizotypal traits showed more errors in response to incongruent 
words than did controls. The control group showed significantly greater FN amplitude in response to incongruent stimuli than to congruent stimuli, whereas the schizotypal-trait group did not show significant difference in FN amplitude between incongruent and congruent stimuli. Furthermore, the schizotypal-trait group showed decreased activation of the left cingulate gyrus and medial/ middle frontal gyrus in different waveforms for the incongruent-congruent conditions at $300-400 \mathrm{~ms}$ after stimulus onset. The two groups did not differ in terms of SP amplitudes. These results indicate that individuals with schizotypal traits have difficulties in conflict detection and cognitive inhibition mediated by the cingulate gyrus and PFC.

\section{ACKNOWLEDGEMENTS}

This work was supported by the Korea Science and Engineering Foundation (KOSEF) grant funded by the Korea government (MOST), 20100029281 .

\section{REFERENCES}

[1] Besnier, N., Richard, F., Zendjidjian, X., Kalsdjian, A., Mazzola-Pomietto, P., Adida, M. and Azorin, J.M. (2009) Stroop and emotional Stroop interference in unaffected relative of patients with schizophrenic and bipolar disorders: distinct markers of vulnerability? World Journal of Biological Psychiatry, 10, 809-818. doi:10.1080/15622970903131589

[2] Zalla, T., Joyce, C., Szoke, A., Schurhoff, F., Pillon, B., Komano, O., Perez-Diaz, F., Bellivier, F., Alter, C., Dubois, B., Rouillon, F., Houde, O. and Leboyer, M. (2004) Executive dysfunctions as potential markers of familial vulnerability to bipolar disorder and schizophrenia. Psychiatry Research, 121, 207-217. doi:10.1016/S0165-1781(03)00252-X

[3] Stroop, J. R. (1935) Studies of interference in serial verbal reaction. Journal of Experimental Psychology, 18, 643-662. doi:10.1037/h0054651

[4] MacLeod, C.M. (1991) Half a century of research on the Stroop effect: An integrative review. Psychological Bulletin, 109, 163-203. doi:10.1037//0033-2909.109.2.163

[5] Carter, C.S., Robertson, L.C. and Nordahl, T.E. (1992) Abnormal processing of irrelevant information in chronic schizophrenia: Selective enhancement of Stroop facilitation. Psychiatry Research, 41, 137-146. doi:10.1016/0165-1781(92)90105-C

[6] Buchanan, R.W., Strauss, M.E., Kirkpatrick, B., Holstein, C., Breier, A. and Carpenter, W.T.J. (1994) Neuropsychological impairments in deficit vs. nondeficit forms of schizophrenia. Archives of General Psychiatry, 51, 804811.

[7] Carter, C.S., Mintun, M., Nichols, T. and Cohen, J.D. (1997) Anterior cingulate gyrus dysfunction and selective attention deficits in schizophrenia: $\left[{ }^{15} \mathrm{O}\right] \mathrm{H}_{2} \mathrm{O}$ PET study during single-trial Stroop task performance. American
Journal of Psychiatry, 154, 1670-1675.

[8] Chen, E.Y.H., Wong, A.W.S., Chen, R.Y.L. and Au, J.W.Y. (2001) Stroop interference and facilitation effects in first-episode schizophrenic patients. Schizophrenia Research, 48, 29-44. doi:10.1016/S0920-9964(00)00107-9

[9] Barch, D.M., Carter, C.S., Perlstein, W., Baird, J., Cohen, J.D. and Schooler, N. (1999) Increased Stroop facilitation effects in schizophrenia are not due to increased automatic spreading activation. Schizophrenia Research, 39, 51-64. doi:10.1016/S0920-9964(99)00025-0

[10] Kerns, J.G., Cohen, J.D., MacDonald, A.W., Cho, R.Y., Stenger, V.A. and Carter, C.S. (2004) Anterior cingulate conflict monitoring and adjustments in control. Science, 303, 1023-1026. doi:10.1126/science. 1089910

[11] Leung, H.C., Skudlarski, P., Gatenby, J.C., Peterson, B. and Gore, J.C. (2000) An event-related functional MRI study of the Stroop color words interference task. Cerebral Cortex, 10, 552-560. doi:10.1093/cercor/10.6.552

[12] Ungar, L., Nestor, P.G., Niznikiewicz, M.A., Wible, C.G. and Kubicki, M. (2010) Color Stroop and negative priming in schizophrenia: An fMRI study. Psychiatry Research: Neuroimaging, 181, 24-29. doi:10.1016/j.pscychresns.2009.07.005

[13] Leon-Carrion, J., Damas-Lopez, J., Martin-Rodriguez, J.F., Dominguez-Roldan, J.M., Murillo-Cabezas, F., BarrosoY-Martin, J.M. and Dominguez-Morales, M.R. (2008) The hemodynamics of cognitive control: The level of concentration of oxygenated hemoglobin in the superior prefrontal cortex varies as a function of performance in a modified Stroop task. Behavioural Brain Research, 193, 248-256.

[14] Schroeter, M.L., Zysset, S., Kupka, T., Kruggel, F. and Yves von Cramon, D. (2002) Near-infrared spectroscopy can detect brain activity during a color-word matching Stroop task in an event-related design. Human Brain Mapping, 17, 61-71. doi:10.1002/hbm.10052

[15] Melcher, T., Falkai, P. and Gruber, O. (2008) Functional brain abnormalities in psychiatric disorders: Neural mechanisms to detect and resolve cognitive conflict and interference. Brain Research Review, 59, 96-124 doi:10.1016/j.brainresrev.2008.06.003

[16] Nordahl, T.E., Carter, C.S., Salo, R.E., Kraft, L., Baldo, J., Salamat, S., Robertson, L. and Kusubov, N. (2001) Anterior cingulate metabolism correlates with Stroop errors in paranoid schizophrenia patients. Neuropsychopharmacology, 25, 139-148. doi:10.1016/S0893-133X(00)00239-6

[17] Yucel, M., Pantelis, C., Stuart, G.W., Wood, S.J., Maruff, P., Velakoulis, D., Pipingas, A., Crowe, S.F., TochonDanguy, H.J. and Egan, G.F. (2002) Anterior cingulate activation during Stroop task performance: A PET to MRI coregistration study of individual patients with schizophrenia. American Journal of Psychiatry, 159, 251-254. doi:10.1176/appi.ajp.159.2.251

[18] Takei, K., Yamasue, H., Abe, O., Yamada, H., Inoue, H., Suga, M., Muroi, M., Sasaki, H., Aoki, S. and Kasai, K. (2009) Structural disruption of the dorsal cingulum bundle is associated with impaired Stroop performance in patients with schizophrenia. Schizophrenia Research, 114, 


\section{9-127. doi:10.1016/i.schres.2009.05.012}

[19] Liotti, M., Woldorff, M.G., Perez III, R. and Mayberg, H.S. (2000) An ERP study of the temporal course of the Stroop color-word interference effect. Neuropsychologia, 38, 701-711. doi:10.1016/S0028-3932(99)00106-2

[20] Hanslmayr, S., Pastotter, B., Bauml, K.H., Gruber, S., Wimber, M. and Klimesch W. (2008) The electrophysiological dynamics of interference during the Stroop task. Journal of Cognitive Neuroscience, 20, 215-224. doi:10.1162/jocn.2008.20.2.215

[21] West, R. and Alain, C. (2000) Effects of task context and fluctuation of attention on neural activity supporting performance of the Stroop task. Brain Research, 873, 102111. doi:10.1016/S0006-8993(00)02530-0

[22] Markela-Lerenc, J., Schmidt-Kraepelin, C., Roesch-Ely, D., Mundt, C., Weisbrod, M. and Kaiser, S. (2009) Stroop interference effect in schizophrenic patients: An electrophysiological approach. International Journal of Psychophysiology, 71, 248-257.

doi:10.1016/j.ijpsycho.2008.10.005

[23] McNeely, H.E., Christensen, B.K., West, R. and Alain, C. (2003) Changes in neurophysiological correlates of conflict processing precede behavioral disturbance in patients with schizophrenia. Journal of Abnormal Psychology, 112, 679-688.

[24] Badzakova-Trajkov, G., Barnett, K.J., Waldie, K.E. and Kirk, I.J. (2009) An ERP investigation of the Stroop task: The role of the cingulate in attentional allocation and conflict resolution. Brain Research, 1253, 139-148. doi:10.1016/j.brainres.2008.11.069

[25] West, R. (2003) Neural correlates of cognitive control and conflict detection in the Stroop and digit-location tasks. Neuropsychologica, 41, 1122-1135. doi:10.1016/S0028-3932(02)00297-X

[26] Markela-Lerenc, J., Ille, N., Kaiser, S., Fiedler, P., Mundt, C. and Weisbrod, M. (2004). Prefrontal-cingulate activation during executive control: Which comes first? Cognitive Brain Research, 18, 278-287. doi:10.1016/j.cogbrainres.2003.10.013

[27] West, R., Bowry, R. and McConville, C. (2004) Sensitivity of medial frontal cortex to response and nonresponse conflict. Psychophysiology, 41, 739-748. doi:10.1111/j.1469-8986.2004.00205.x

[28] West, R., Perry, M., Moore, K., Jakubek, K. and Wymbs, N. (2005) Neural correlates of conflict processing. Experimental Brain Research, 873, 38-48. doi:10.1007/s00221-005-2366-y

[29] Pascual-Marqui, R.D., Michel, C.M. and Lehmann D. (1994) Low resolution electromagnetic tomography: A new method for localization electrical activity in the brain. International Journal of Psychophysiology, 18, 49-65. doi:10.1016/0167-8760(84)90014-X

[30] Cuffin, B.N. (1998) EEG dipole source localization. Engineering in Medicine \& Biology Magazine, IEEE, 17, 118-122. doi:10.1109/51.715495

[31] Siever, L.J. and Davis, K.L. (2004) The pathophysiology of schizophrenia disorders: Perspectives from spectrum. American Journal of Psychiatry, 161, 398-413. doi:10.1176/appi.ajp.161.3.398

[32] Lin, H.F., Liu, Y.L., Liu, C.M., Hung, S.I., Hwu, H.G. and Chen W.J. (2005) Neuregulin I gene and variations in perceptual aberration of schizotypal personality in adolescents. Psychological Medicine, 35, 1589-1598. doi:10.1017/S0033291705005957

[33] Moorhead, T.W.J., Stanfield, A., Spencer, M., Hall, J., McIntosh, A., Qwnes, D.C., Lawrie, S. and Johnstone, E. (2009) Progressive temporal lobe gray matter loss in adolescents with schizotypal traits and mild intellectual impairment. Psychiatry Research: Neuroimaging, 174, 105-109. doi:10.1016/j.pscychresns.2009.04.003

[34] Noguchi, H., Hori, H. and Kunugi, H. (2008) Schizotypal traits and cognitive function in healthy adults. Psychiatry Research, 161, 162-169. doi:10.1016/j.psychres.2007.07.023

[35] Cadenhead, K.S., Perry, W., Shafer, K. and Braff, D.L. (1999) Cognitive function in schizotypal personality disorder. Schizophrenia Research, 37, 123-132. doi:10.1016/S0920-9964(98)00147-9

[36] Orem, D.M. and Bedwell, J.S. (2010) A preliminary investigation on the relationship between color-word Stroop task performance and delusion-proneness in nonpsychiatric adults. Psychiatry Research, 175, 27-32. doi:10.1016/j.psychres.2008.09.001

[37] Kaplan, O. and Lubow, R.E. (2011) Ignoring irrelevant stimuli in latent inhibition and Stroop paradigm: The effects of schizotypy and gender. Psychiatry Research, 186, 40-45. doi:10.1016/j.psychres.2010.07.025

[38] Moon, H.O., Yang, I.H., Lee, H.P., Kim, M.E. and Ham, W. (1997) The preliminary study on the validation of schizotypal personality questionnaire-Korean version. Journal of Korean Neuropsychiatry Association, 36, 329-343.

[39] Raine, A. (1992) The SPQ: A scale for the assessment of schizotypal personality based on DSN-III-R criteria. Schizophrenia Bulletin, 17, 555-564.

[40] First, M.B., Spitzer, R.L., Gibbson, M. and Williams, J.B.W. (1996) Structured Clinical Interview for DSM-IV Axis I Disorder. New York State Psychiatric Institute, New York.

[41] Tucker, D.M. (1993) Spatial sampling of head electrical fields: The geodesic sensor net. Electroencephalography and Clinical Neurophysiology, 87, 154-163. doi:10.1016/0013-4694(93)90121-B

[42] Dien, J. (1998) Issues in the application of the average reference: Review, critiques, and recommendations. Behavior Research Methods, Instruments, \& Computers, $\mathbf{3 0}$, 34-43. doi:10.3758/BF03209414

[43] Pascual-Marqui, R.D. (2002) Standardized low-resolution brain electromagnetic tomography (sLORETA). Experimental and Clinical Pharmacology, 24, 5-12.

[44] Park, H.J., Kwon, J.S., Youn, T., Pae, J.S., Kim, J.J., Kim, M.S. and Ha, K.S. (2002) Statistical parametric mapping of LORETA using high density EEG and individual MRI: Application to mismatch negativities in schizophrenia. Human Brain Mapping, 17, 168-178. doi:10.1002/hbm.10059

[45] Barch, D.M., Carter, C.S. and Cohen, J.D. (2004) Factors 
influencing Stroop performance in schizophrenia. Neuropsychology, 18, 477-484.

doi:10.1037/0894-4105.18.3.477

[46] Henik, A. and Salo, R. (2004) Schizophrenia and the Stroop effect. Behavior and Cognitive Neuroscience Review, 3, 42-59. doi:10.1177/1534582304263252

[47] Hepp, H.H., Maier, S., Hermle, L. and Spitzer, M. (1996) The Stroop effect in schizophrenic patients. Schizophrenia Research, 22, 187-195. doi:10.1016/S0920-9964(96)00080-1

[48] West, R. and Alain, C. (1999) Event-related neural activity associated with the Stroop task. Cognitive Brain Research, 8, 157-164. doi:10.1016/S0926-6410(99)00017-8

[49] Carter, C.S., Robertson, L.C., Nordahl, T.E., O'Shora-Celaya, L.J. and Chaderjian, M.C. (1993) Abnormal processing of irrelevant information in schizophrenia: The role of illness subtype. Psychiatry Research, 48, 17-26.
doi:10.1016/0165-1781(93)90109-T

[50] Albanese, A.M., Merlo, A.B., Mascitti, T.A., Tornese, E.B., Gomez, E.E., Konopka, V. and Albanese, E.F. (1995) Inversion of the hemispheric laterality of the anterior cingulate gyrus in schizophrenics. Biological Psychiatry, 38, 13-21. doi:10.1016/0006-3223(94)00257-4

[51] Pantelis, C., Yucel, M., Wood, S.J., McGorry, P. and Velakoulis, P. (2001) The timing and functional consequences of structural brain abnormalities in schizophrenia. Neuroscience News, 4, 36-46.

[52] Takahashi, T., Suzuki, M., Kawasaki, Y., Kurokawa, K., Hagino, H., Yamashita, I., Zhou, S.Y., Nohara, S., Nakamura, K., Seto, H. and Kurachi, M. (2002) Volumetric magnetic resonance imaging study of the anterior cingulate gyrus in schizotypal disorder. European Archives of Psychiatry and Clinical Neurosciences, 252, 268-277. doi:10.1007/s00406-002-0392-3 